\title{
ALTERNATIVA QUIRÚRGICA DE REPARACIÓN DUODENAL EN PEDIATRÍA (PRESENTACIÓN DE CASO), HOSPITAL INFANTIL DR. ROBERT REID CABRAL (HIRRC), REPÚBLICA DOMINICANA
}

\author{
Surgical alternative of duodenal repair in pediatrics \\ (presentation of case), Robert Reid Cabral Children's Hospital, \\ Dominican Republic
}

\author{
Carlos Cabrera Valerio ${ }^{a}$, Firelys Roa ${ }^{b}$, Rosa Castilloc, Laiz Decamps ${ }^{\mathrm{d}}$ y Luis Feliz
}

Recibido: 3 de marzo, $2020 \bullet$ Aprobado: 6 de junio, 2020

Cómo citar: Cabrera Valerio C, Roa F, Castillo R. Alternativa quirúrgica de reparación duodenal en pediatría (presentación de caso), Hospital Infantil Dr. Robert Reid Cabral (HIRRC), República Dominicana. cysa [Internet]. 15 de febrero de 2021 [citado 23 de febrero de 2021];5(1):129-36. Disponible en: https://revistas.intec.edu.do/index.php/cisa/article/view/2053

\section{Resumen}

Introducción: el trauma cerrado de abdomen en pediatría ocasiona lesión de órganos abdominales en $8-12 \%$ de la población, siendo lesiones duodenales solo el $10 \%$ de estas, por lo que son poco frecuentes en pediatría. La principal causa de lesión duodenal se relaciona con abuso infantil, siendo los accidentes de vehículos de motor el segundo mecanismo más común. Cuando se trata de lesiones no destructivas, la reparación primaria del duodeno es razonable. Sin embargo, las lesiones destructivas ameritan estrategias de reparación más complejas.

Material y Método: presentamos paciente masculino de tres años edad con lesión duodenal grado III en ángulo duodenoyeyunal, la misma fue intervenida inicialmente mediante anastomosis primaria, presentado posterior dehiscencia. En su segunda intervención se realiza cierre duodenal distal y anastomosis gastroyeyunal, favoreciendo flujo retrogrado de vías biliares a estómago, con adecuada evolución.

\footnotetext{
a Cirujano Pediatra del Hospital Infantil Dr. Robert Reid Cabral (HIDRRC), Santo Domingo, República Dominicana. ORCID: 0000-0003-2576-3912, Correo-e: cirugia@hirrc.gov.do

b Cirujana Pediatra del Hospital Infantil Dr. Robert Reid Cabral (HIDRRC), Santo Domingo, República Dominicana.

Correo-e: cirugia@hirrc.gov.do

c Residente de Cirugía Pediátrica del Hospital Infantil Dr. Robert Reid Cabral (HIDRRC), Santo Domingo, República Dominicana.

Correo-e: cirugia@hirrc.gov.do
}

\begin{abstract}
Introduction: Blunt trauma of the abdomen in pediatrics causes injury to abdominal organs in 8-12\% of the population, with duodenal injuries being only $10 \%$ of these, which is why they are rare in pediatrics. The main cause of duodenal injury is related to childhood abuse, with motor vehicle accidents being the second most common mechanism. When it comes to nondestructive injury, primary duodenal repair is reasonable. However, destructive injuries warrant more complex repair strategies.
\end{abstract}

Material and Method: We present a 3-year-old male patient with a grade III duodenal injury at the duodenojejunal angle, which was initially operated on by means of a primary anastomosis, with subsequent dehiscence. In his second operation, a distal duodenal closure and a gastrojejunal anastomosis were performed, favoring retrograde flow of the bile ducts to the stomach, with adequate evolution.

\footnotetext{
d Residente de Cirugía Pediátrica del Hospital Infantil Dr. Robert Reid Cabral (HIDRRC), Santo Domingo, República Dominicana. Correo-e: cirugia@hirrc.gov.do

e Residente de Cirugía Pediátrica del Hospital Infantil Dr. Robert Reid Cabral (HIDRRC), Santo Domingo, República Dominicana. Correo-e: cirugia@hirrc.gov.do
} 
Conclusión: existen múltiples abordajes quirúrgicos, los cuales representan aun hoy gran morbilidad. El cierre duodenal con la posterior decisión de cómo derivar las vías biliares, es probablemente el mayor desafío. Se plantea la posibilidad del cierre duodenal distal a las vías biliares, con drenaje retrógrado. Por lo cual la gastroyeyunostomía es una técnica alternativa en pediatría en la reparación de lesiones complejas del duodeno.

Palabras clave: lesión duodenal; trauma duodenal; reparación duodenal; gastroyeyunostomía; técnicas en pediatría.

\section{Introducción}

El manejo quirúrgico de las lesiones duodenales destructivas representa un reto para todo cirujano. Las técnicas existentes y que ha sido descritas, generalmente envuelven procedimientos agresivos con reparación de la lesión, derivación de las vías biliares y drenajes para control de secreciones del duodeno y el páncreas. ${ }^{1}$

El trauma cerrado de abdomen en pediatría ocasiona lesión de órganos abdominales en 8-12\% de la población, siendo lesiones duodenales solo el $10 \%$ de estas, por lo que son poco frecuentes en pediatría. $^{2}$

La principal causa de lesión duodenal se relaciona con abuso infantil, sobre todo en menores de cuatro años, siendo los accidentes de vehículos de motor el segundo mecanismo más común. Cuando se trata de lesiones no destructivas, la reparación primaria del duodeno es razonable. Sin embargo, las lesiones destructivas ameritan estrategias de reparación más complejas. $^{3}$

El objetivo de esta publicación es dar a conocer un abordaje alternativo de los ya descritos, respetando los principios quirúrgicos de la reparación de lesiones duodenales. De acuerdo con el contenido de la literatura, es evidente que existe la necesidad del fomento de abordajes, que disminuyan la morbi-
Conclusion: There are multiple surgical approaches, which still represent great morbidity today. Duodenal closure with the subsequent decision on how to bypass the bile ducts is probably the greatest challenge. The possibility of duodenal closure distal to the bile ducts, with retrograde drainage, is raised. Therefore, gastrojejunostomy is an alternative technique in pediatrics for the repair of complex duodenal injuries.

Keywords: Duodenal lesions; duodenal trauma; duodenal repair; gastrojejunostomy; pediatric techniques.

lidad relacionada con los procedimientos. A continuación, presentamos un caso de lesión duodenal grado III en el ángulo duodenoyeyunal.

\section{Reporte de caso}

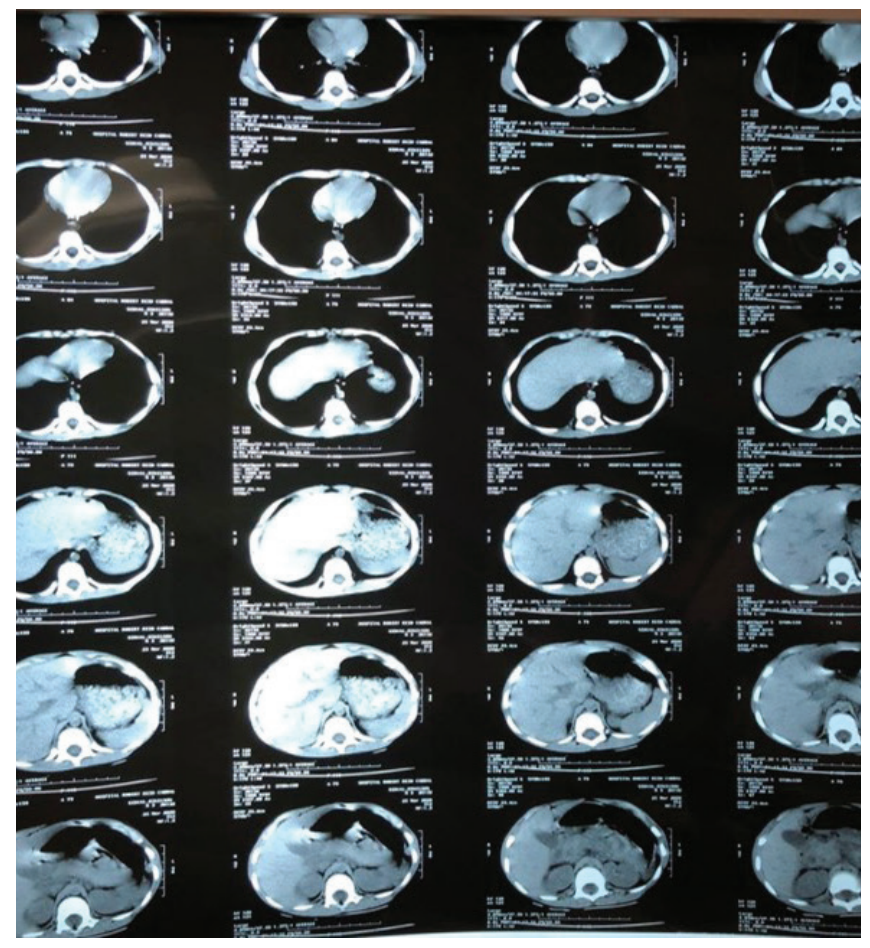

Figura 1: Tomografía abdominal donde se observa líquido hipodenso periduodenal, pancreático y perihepático

Fuente: Banco de imágenes del Servicio de Cirugía pediátrica Hospital Dr. Robert Reid Cabral. 


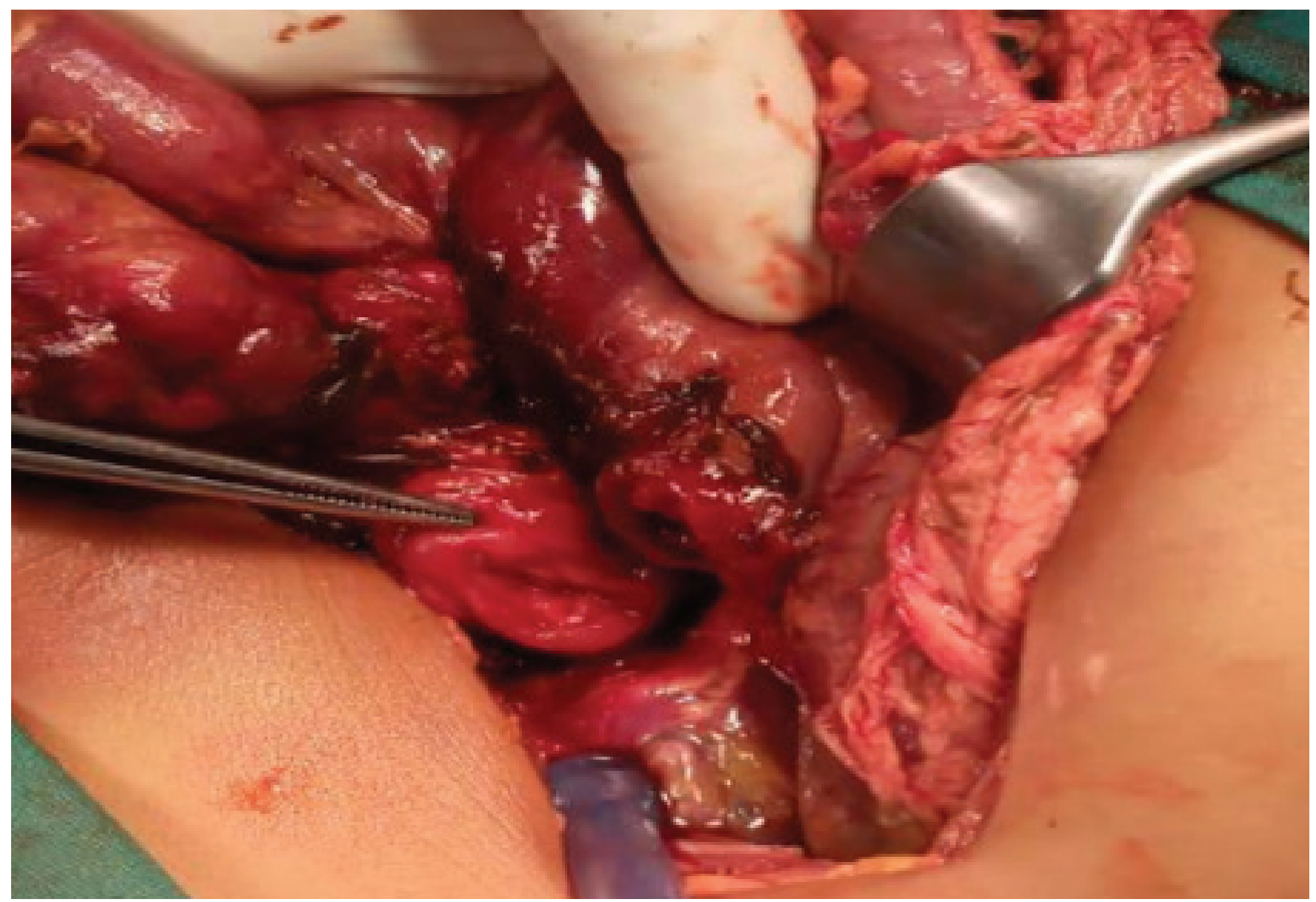

Figura 2. Esta imagen muestra la rotura (disrupción) del duodeno señalado por la flecha

Fuente: Banco de imágenes del Servicio de Cirugía pediátrica Hospital Dr. Robert Reid Cabral.

Masculino de tres años de vida, quien acude con historia de 4 hrs previas a su llegada fue impactado por motocicleta. Al examen físico paciente se encontraba con signos vitales dentro de parámetros de referencia para edad y sexo. Presentaba abdomen semigloboso, depresible, doloroso a la palpación difusa, sin signos de irritación peritoneal en ese momento, peristalsis presente, masa que protruye en región umbilical, reducible, no dolorosa de bordes libres, defecto de 1 $\mathrm{cm}$ aproximadamente. Quemaduras por fricción en cuero cabelludo (región occipital) y miembro inferior derecho, herida cortante en rodilla derecha suturada. Sus analíticas de ingreso reportan Hemoglobina 10.8 mg/dL, Hematocrito 31 \%, Plaquetas 381,000 mm3. Enzimas hepáticas: Transaminasa glutámica oxalacética 338 U/L, Transaminasa glutámica pirú- vica $134 \mathrm{U} / \mathrm{L}$, Gamma glutamil transferasa $23 \mathrm{U} / \mathrm{L}$. Amilasa 208 U/L, Lipasa 117 U/L.

Se realiza sonografía abdominal que reporta bazo de aspecto heterogéneo asociado a moderada cantidad de líquido hacia polo inferior, a favor de probable lesión esplénica. Líquido libre en ambas fosas ilíacas.

Se realiza TAC abdominal donde se observa zona de heterogenicidad hacia la topografía de la 3ra y 4 ta porción duodenal con líquido (imagen hipodenso) a este nivel, también discreta hipodensidad (líquido) peri-hepática y peri-pancreática (figura1).

Tras presentar datos de irritación peritoneal aproximadamente 12 hrs después, se decide realizar 
laparotomía exploratoria, donde se evidencia disrupción total del ángulo duodeno-yeyunal (figura 2). Inicialmente se realiza verticalización del duodeno, resección y anastomosis primaria duodeno-yeyunal + gastrostomía por técnica de Stamm.

Paciente presentó evolución tórpida al 9no día con abdomen doloroso y signos de irritación peritoneal más salida abundante de secreciones por sitio quirúrgico. Se lleva a cirugía (reintervención), evidenciando dehiscencia de las fascias de la pared abdominal, desperitonización de la gastrostomía más dehiscencia del $90 \%$ de la anastomosis primaria, abscesos interasa y adherencias múltiples intestinales. Se procedió a realizar en esta ocasión cierre duodenal distal en bolsa ciega + gastroyeyunostomía (anastomosis gastroyeyunal) (véase figura 3), en vista de que otra anastomosis duodeno-yeyunal quedaría con mucha tensión; también todo el intestino se encontraba inflamado con muchas serositis. Factores a considerar a la hora de hacer una anastomosis. Previa realización de la gastroyeyunostomía se confirmó salida de bilis retrograda hacia estómago. Además, se colocó un drenaje de saratoga.

Paciente se mantuvo recibiendo soporte nutricional parenteral, y a las 72 horas presentó disminución del débito por sonda nasogástrica, evacuaciones verdosas que traduce el paso de contenido biliar por el tracto gastrointestinal por lo cual se inicia la vía oral al 5 to día; y es egresado satisfactoriamente al 10mo día de su reintervención.

\section{Discusión}

El hematoma duodenal, puede manejarse conservadoramente; mientras que, en casos de perforación duodenal, se necesita reparación quirúrgica. ${ }^{4,5,6}$

Las lesiones duodenales destructivas son de difícil abordaje debido a las grandes intervenciones que en sentido general requieren, y han representado un paradigma en las últimas décadas. ${ }^{7}$

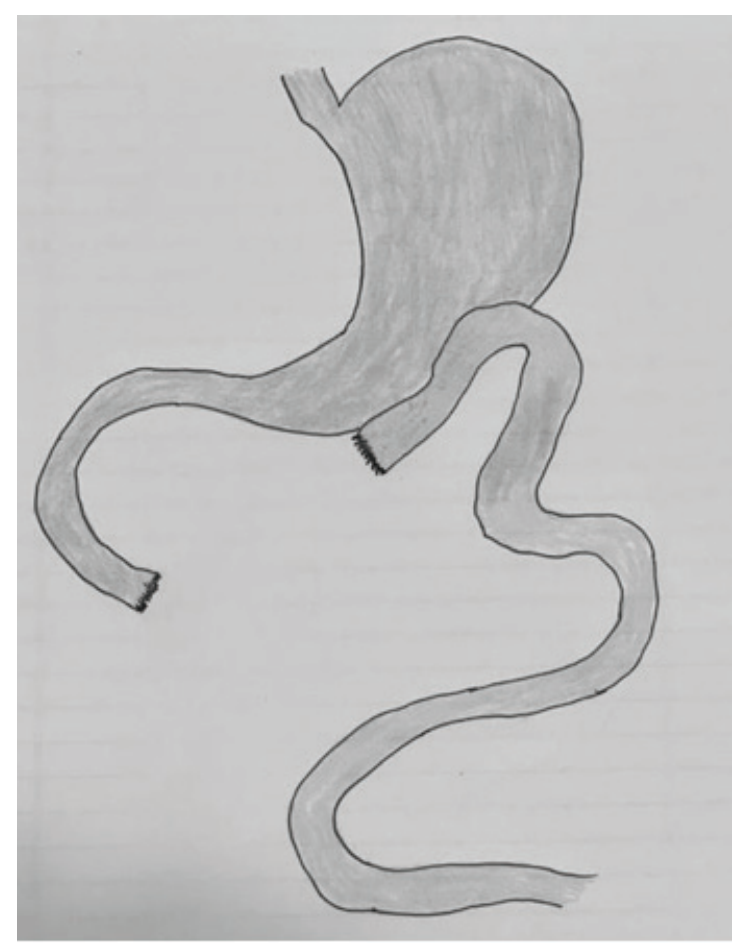

Figura 3: Esquema de la gastroyeyunostomía con cierre duodenal distal en bolsa ciega, con drenaje de la vía biliar de forma retrograda. Flecha roja estómago, flecha verde duodeno, flecha azul yeyuno.

La estadificación de la lesión, no nos orienta hacia la decisión de una técnica sobre otra, más bien nos habla en términos pronósticos. Sin embargo, suele ser óptimo estadificarla bajo la lógica de que todas las lesiones grado III en adelante, entran dentro de la designación de complejas, independientemente de la porción anatómica del duodeno lesionado ${ }^{8}$, (tabla 1). En ese sentido, la orientación comprende varios abordajes, siempre que sea posible, se recomienda la reparación primaria de las lesiones. ${ }^{8,9}$

Tras realizar la laparotomía, se deben controlar inicialmente la hemorragia y la contaminación gastrointestinal. ${ }^{9}$ Se puede reparar la lesión mediante una duodenorrafia. La decisión de realizarla está a disposición del cirujano, sin embargo, hay literaturas que sugieren reparar siempre y cuando la lesión sea $<$ de $3 \mathrm{cms}$. Además de esta, la reparación se puede realizar mediante resección y anastomosis (duodeno-duodeno o duodeno-yeyuno). ${ }^{10}$ 


\section{Alternativa quirúrgica de reparación duodenal en pediatría (resentación de caso), Hospital Infantil \\ Dr. Robert Reid Cabral (HIRRC), República Dominicana}

Tabla 1. Escakak de clasificación de lesiones duodenales

\begin{tabular}{lll}
\hline Grado & Lesion & Descripción \\
\hline II & $\begin{array}{l}\text { Hematoma } \\
\text { Laceracion }\end{array}$ & $\begin{array}{l}\text { Afectación de una única porción duodenal } \\
\text { Laceración parcial sin perforación. }\end{array}$ \\
\hline III & $\begin{array}{l}\text { Hematoma } \\
\text { Laceracion }\end{array}$ & $\begin{array}{l}\text { Afectación de más de una porción duodenal. } \\
\text { Disrupción de }<50 \% \text { de la circunferencia duodenal. }\end{array}$ \\
\hline Laceracion & $\begin{array}{l}\text { Disrupción de }<50 \% \text { al } 75 \% \text { de la circunferencia de la } \\
\text { segunda porción duodenal. O Disrupción de }<5 \% \\
\text { al } 100 \% \text { de la circunferencia de la primera, tercera y } \\
\text { cuarta porciones duodenales. }\end{array}$ \\
\hline Laceracion & $\begin{array}{l}\text { Disrupción de }>75 \% \text { de la circunferencia de la segunda } \\
\text { porción duodenal. Afectación de la ampolla de váter o } \\
\text { de la porción distal del conducto coledoco. }\end{array}$ \\
\hline Fuente: Organ Injury Scaling Committee de la American Association for the Surgeryof Trauma
\end{tabular}

Cualquiera que sea el caso (rafia o resección y anastomosis), puede ser necesario dejar un drenaje protector de la reparación; además, en casos más especiales se coloca un tubo de duodenostomía. Siempre es idónea la colocación de un drenaje, constituyéndolo una fístula, antes que un cierre precario. En contraste se describen desventajas en cuanto al uso de tubos de duodenostomía, ya que estas fístulas, generalmente, son de gasto alto y muy dificiles de cerrar. Resulta controversial la colocación de este tubo, pues existen tres formas de construcción, ninguna de las cuales parece ser más beneficiosa que la otra: duodenostomía primaria, que consiste en colocar un tubo cuyo drenaje se proyecte hacia la pared abdominal a través de un orificio diferente al de la herida; duodenostomía anterograda, que consiste en la colocación de un tubo hacia duodeno a través del píloro; y duodenostomía retrógrada, en donde se coloca un tubo hacia duodeno desde una yeyunostomía. ${ }^{11}$

En casos en donde la lesión sea lo suficientemente comprometedora, y no sea factible realizar una rafia de duodeno o resección y anastomosis, entonces se recomienda cierre duodenal. En ese mismo orden las técnicas descritas son las siguientes:

La técnica de los tres tubos, que consiste en descomprirmir el duodeno luego de reparado, en sentido retrógrado desde el yeyuno (yeyunostomía retro- grada); habilitar una vía de alimentación a través del sondaje anterógrado del yeyuno; $y$, por último, construir una gastrostomía con la finalidad de descomprimir el estómago. ${ }^{2,3,8}$

Otra opción corresponde con la técnica de Berne (diverticulación del duodeno), que consiste en realizar un cierre duodenal y duodenostomía descompresiva; coledocotomía o cistotomía con la finalidad de drenar las vías biliares; gastrostomía de alimentación y gastroyeyunostomía (resección de antro y vagotomía). ${ }^{8}, 9$ Esta técnica ha perdido auge en las últimas décadas debido a la complejidad de su construcción, además dentro de sus desventajas plantea resecar un antro gástrico previamente sano. ${ }^{12}$

La exclusión pilórica o técnica de Jordan, se puede integrar en cualquiera de estas técnicas, según sea la necesidad, con la finalidad de derivar las secreciones gástricas. ${ }^{2,5,8}$

La duodenopancreatectomía o procedimiento de Whipple, generalmente se reserva para lesiones destructivas de la 2 da porción del duodeno, la incapacidad de reconstruir la vía biliar ${ }^{4}$, o cuando ha habido un compromiso importante de la vascularidad duodeno-pancreática, ${ }^{2,10}$. Esta contempla la resección amplia de cabeza páncreas, duodeno, vesícula biliar, conducto biliar distal, yeyuno proximal y el antro gástrico. ${ }^{13}$ 
A pesar de las distintas técnicas descritas, no hay consenso a la hora de utilizar una u otra, en ciruncunstacias específicas, por lo que la decisión sigue siendo del cirujano, de acuerdo a la magnitud de la lesión frente a la que se encuentre.

En términos generales cualquiera sea la técnica que se vaya a utilizar, se deben respetar los siguientes principios quirúrgicos, ya que contribuyen a menor morbilidad y reducción de la estadía:

- Siempre que exista la posibilidad priorizar el cierre primario

- Establecer una vía de drenaje segura para las vias biliares

- Asegurar una vía de alimentación óptima

- Asegurar la descompresión gástrica, tras el cierre duodenal. ${ }^{2,3,7,8}$

Nuestro paciente tuvo una lesión inicial de la unión duodeno yeyunal que experimentó necrosis parcial, por lo que se resecó la 4ta y parte de la 3ra porción del duodeno. En ese sentido se plantea la disyuntiva de como derivar las vías biliares. Por lo que en lugar de realizar cierre duodenal, proximal a las vías biliares, fue preferible respetar la viabilidad del duodeno y realizar cierre distal a las vías biliares, con la finalidad de drenarlas en sentido retrógrado (hacia estómago). Se realizó gastroyeyunostomía, sin recurrir a la resección del antro gástrico o vagotomía, descritas universalmente.

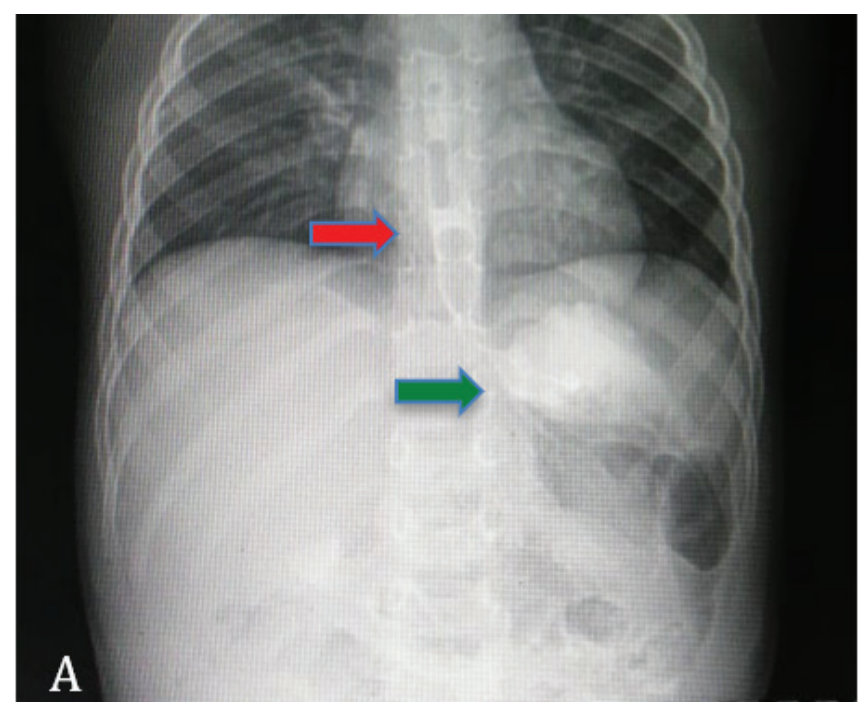

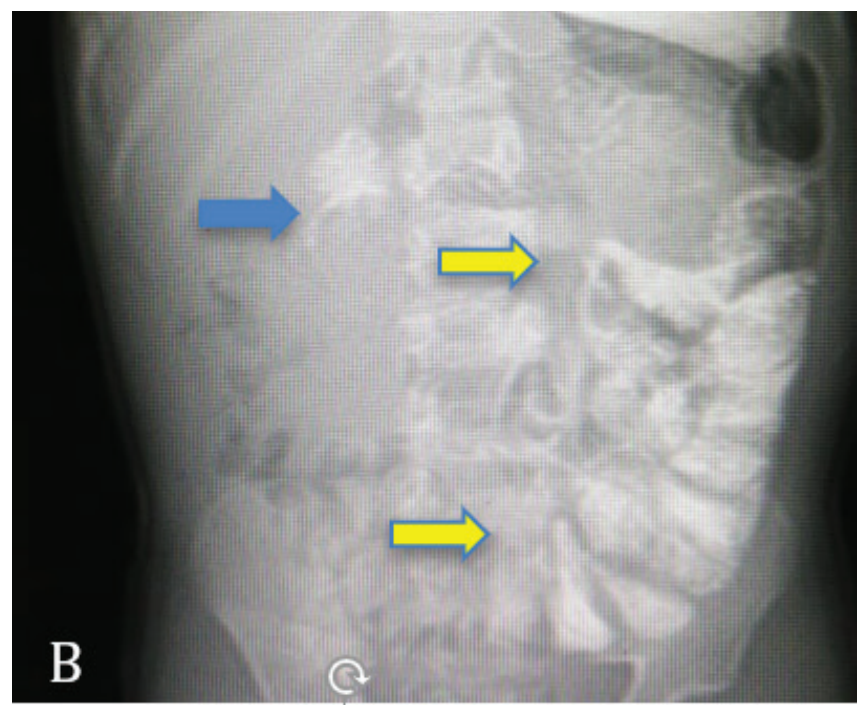

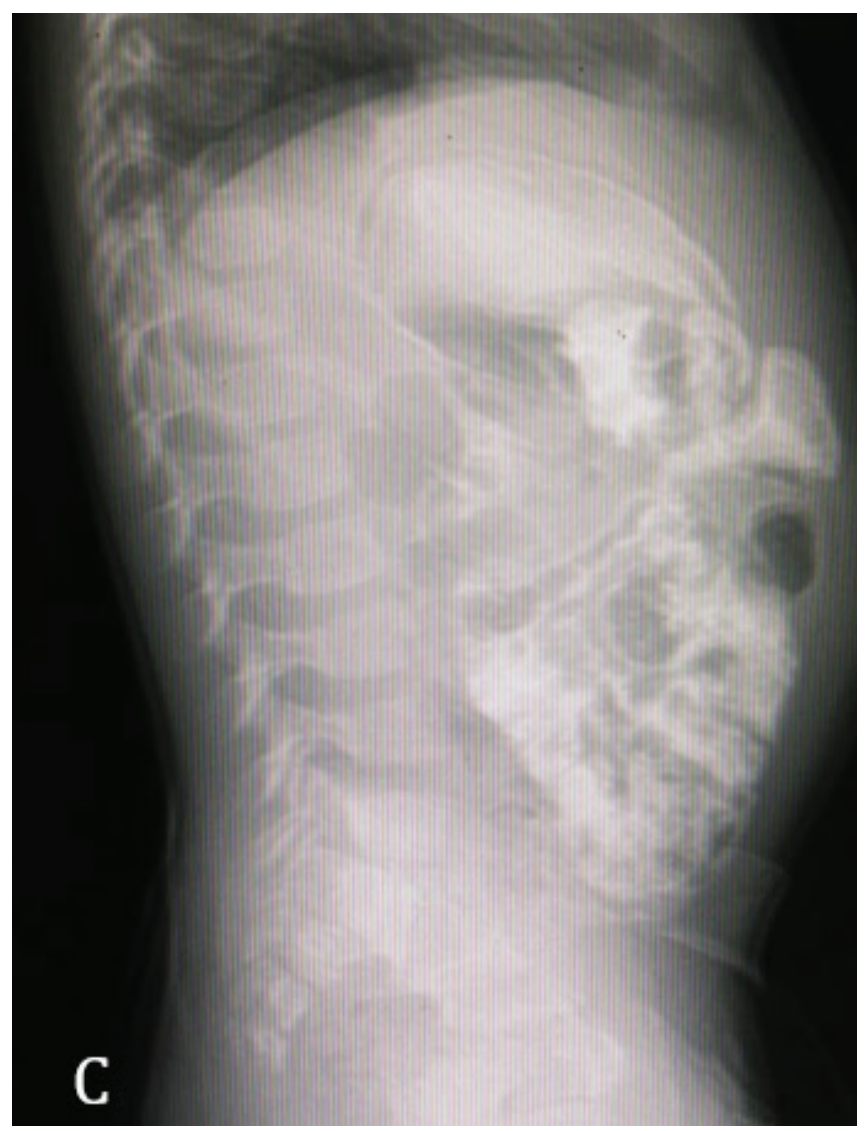

Figura 4: -A. Se observa el esófago flecha roja y estómago fecha verde, constrastado al minuto de ingerirlo, medio hidrosoluble. -B. Se observa a la hora contraste en estómago; flecha azul indica el duodeno y flechas amarillas el yeyunoileon. -C. Una vista lateral donde visualiza el paso adecuado del contraste. 
La evolución fue satisfactoria, pese a la realización de una técnica diferente, y se respetaron los princios quirúrgicos previamente descritos.

\section{Conclusión}

Las lesiones duodenales siguen representando un reto para el cirujano, aun más para el cirujano infantil. Existen multiples abordajes quirúrgicos, los cuales representan, aun hoy, gran morbilidad. El cierre duodenal con la posterior decisión de cómo derivar las vías biliares es probablemente el mayor desafio.

Se plantea la posibilidad del cierre duodenal, distal a las vías biliares, con drenaje retrógrado hacia el estómago. Esto elimina la necesidad de yeyunostomía de alimentación, así como la necesidad de un procedimiento quirúrgico posterior para realizar el cierre. Elimina la necesidad también de colocar tubos de duodenostomía, ya que se favorece el drenaje duodenal a pesar de ser retrógrado, evitando así todas las complicaciones que de ella derivan. En efecto, la gastroyeyunostomía es una técnica alternativa en pediatría en la reparación de lesiones complejas del duodeno.

\section{Bibliografía}

1. Magaña Sánchez I J, García Núñez L M Alternativas de manejo del trauma duodenal. Asociación Mexicana de Cirugía General. Cirujano General, 2013;35:Supl. 1.

2. Coran, Arnold G. Pediatric Surgery. "Abdominal Trauma". (7th ed.). Saunders: (C) Elsevier; 2012. p. 289-310.

3. Spitz L, Coran A. Operative Pediatric Surgery. Mark Davenport. (7th ed.); 2013

4. Makhija D, Jayaswal S, Kumbhar V, Shan H. Traumatic duodenal injury in children: A report of two cases. Department of Pediatric Surgery,
TNMC \& BYL Nair Hospital, Mumbai, Maharashtra-India; 2017

5. Clendenon J N, Meyers R L, Nance ML, Escaife ER, Management of duodenal injuries in children. Salt Lake City, Utah and Philadelphia, Pennsylvania. J Pediatric Surg, 2004;39:964-8.

6. Peterson ML, Abbas PI, Fallon SC, Naik-Mathuria BJ, Rodriguez JR. Management of traumatic duodenal hematomas in children. Division of pediatric surgery, Michael E. DeBakey Department of Surgery, Baylor Collegue of Medicine, Houston, Texas; 2015

7. Weale RD, Kong VY, Bekker W, Bruce JL, Oosthuizen GV, Laing GL, Clarke DL. Primary Repair of duodenal injuries: A Retrospective Cohort Study from A Major Trauma Centre In South Africa. Scandinavian Journal of Surgery, 2019;1-5.

8. Ferrada P, et al. "Management of Duodenal Trauma: A Retrospective Review from The Pan American Trauma Society". Journal of Trauma and Acute Care Surgery, 2018;86(3):392-6.

9. Aiolfi A, Matsushima K, Chang G, Bardes J, Strumwasser A, Lam L, Inabal K, Demetriades D. Surgical Trends in the Management of Duodenal Injury. Journal of Gastrointestinal Surgery, 2019;23: 264-9 Doi: 10.1007/s11605018-3964-x

10. Rodríguez García J A, Ponce-Escobedo A N, Pérez-Salazar D A, Sepulveda-Benavides C A, Uvalle-Villagómez R A, Muñoz-Maldonado G E Lesión duodenal en el traumatismo contuso de abdomen. Informe de caso y revisión bibliográfica. Servicio de cirugía general, Hospital Universitario Dr. José Eleuterio González, Monterrey, Nuevo León, México. 
11. Asensio J A, Petrone P, Pardo M, García W M, Karsidag T. Manejo y revisión histórica de las lesiones duodenales. Division of Trauma and Critical Care. Department of Surgery, Los Angeles County and the University of Southern California. LAC+USC Medical Center. Revista chilena de cirugía, 2003;55(4):313-20.

12. Schroeppel T J, Saleem K, Sharpe J P, Magnotti L J, Weinberg J A, Fischer PE, Croce M A, Fabian T C. Penetrating Duodenal Trauma: A 19-year experience. Trauma Acute Care Surg, 2016;80(3):461-5 Doi:10.1097/TA.000 0000000000934
13. Chincarini M, Zamboni G A, Pozzi Mucelli R. Major Pancreatic Resections: normal postoperative findings and complications. di Radiologia, DAI Patologia e Diagnostica, Verona, Italy; 2018 